\title{
Protein Conjugation with Triazolinediones: Switching from a General Tyrosine-Selective Labeling Method to a Highly Specific Tryptophan Bioconjugation Strategy
}

\author{
Klaas W. Decoene, ${ }^{\dagger}$ Kamil Unal, ${ }^{\ddagger}$ An Staes ${ }^{\ominus \Psi}$, Kris Gevaert ${ }^{\ominus}$, Johan M. Winne, ${ }^{\ddagger}$ Annemieke Madder ${ }^{*}$ \\ † Organic and Biomimetic Chemistry Research group OBCR, Department of Organic and Macromolecular Chemistry, \\ Faculty of Sciences, Ghent University, Krijgslaan 281 S4, 9000 Ghent, Belgium \\ ‡Organic Synthesis group, Department of Organic and Macromolecular Chemistry, Faculty of Sciences, \\ Ghent University, Krijgslaan 281 S4, 9000 Ghent, Belgium \\ ${ }^{\ominus}$ VIB Centre for Medical Biotechnology, Technologiepark-Zwijnaarde 75, 9052 Ghent, Belgium \& Department of Bio- \\ molecular Medicine, Ghent University, Technologiepark-Zwijnaarde 75, 9052 Ghent, Belgium
}

${ }^{\Psi}$ VIB core facility , VIB Centre for Medical Biotechnology, Technologiepark-Zwijnaarde 75, 9052 Ghent, Belgium

\begin{abstract}
Selective labeling of tyrosine residues in peptides and proteins can be achieved via a 'tyrosine-click' reaction with triazolinedione reagents (TAD). We have found that tryptophan residues are in fact often also labeled with this reagent. This off-target labeling is only observed at very low levels in protein bioconjugation but remains under the radar due to the low relative abundance of tryptophan compared to tyrosines in natural proteins, and because of the low availability and accessibility of their nucleophilic positions at the solvent-exposed protein surface. Moreover, because TAD-Trp adducts are known to be readily thermoreversible, it can be challenging to detect these physiologically stable but thermally labile modifications using several MS/MS techniques. We have found that fully solvent-exposed tryptophan side chains are kinetically favored over tyrosines under almost all conditions, and this selectivity can even be further enhanced by modifying the pH of the aqueous buffer to effect selective Trp-labeling. This new site-selective bioconjugation method does not rely on unnatural amino acids and has been demonstrated for peptides and for recombinant proteins. Thus, the TAD-Tyr click reaction can be turned into a highly site-specific labeling method for tryptophans.
\end{abstract}

Site-selective protein modification is of utmost importance for many applications from fundamental biology (fluorescent tagging) to therapeutic development (antibody-drug conjugates).1,2,3,4 While amino acid selectivity can be achieved by exploiting the nucleophilic functionalities of e.g. lysines and cysteines, ${ }^{5,6}$ genuine site selectivity depends on their representation density on the protein surface. In this regard, tryptophan (Trp) is an interesting target for native conjugation strategies, with an abundance of just over $1 \%$ in proteins. ${ }^{7}$ Despite the indole side chain not being the most chemically tractable target, several groups have reported methodologies for selective modification of tryptophan in peptides and proteins. ${ }^{8,9,10,11}$ Many of these strategies employ transition metal catalyzed reactions and/or conditions limiting downstream biochemical applications. These reactions are typically alkynylations and $\mathrm{C}-\mathrm{H}$ arylations of the indole. ${ }^{12,13,14,15,16}$ Also, Trp sulfenylation was demonstrated for peptide ligation. ${ }^{17}$ While Francis and coworkers showed rhodium carbenoid-based Trp labeling at mild $\mathrm{pH},{ }^{18}$ this method is dependent on transition metal catalysis and requires long reaction times. An organoradical Trp conjugation was demonstrated on peptides and proteins ${ }^{19}$ and even if the method is devoid of transition metals, it requires acidic conditions and is not compatible with buffers. Very recently, a novel biomimetic approach for the selective conjugation of tryptophan was developed, this method however employs UV irradiation and needs to be performed in absence of oxygen. ${ }^{20}$

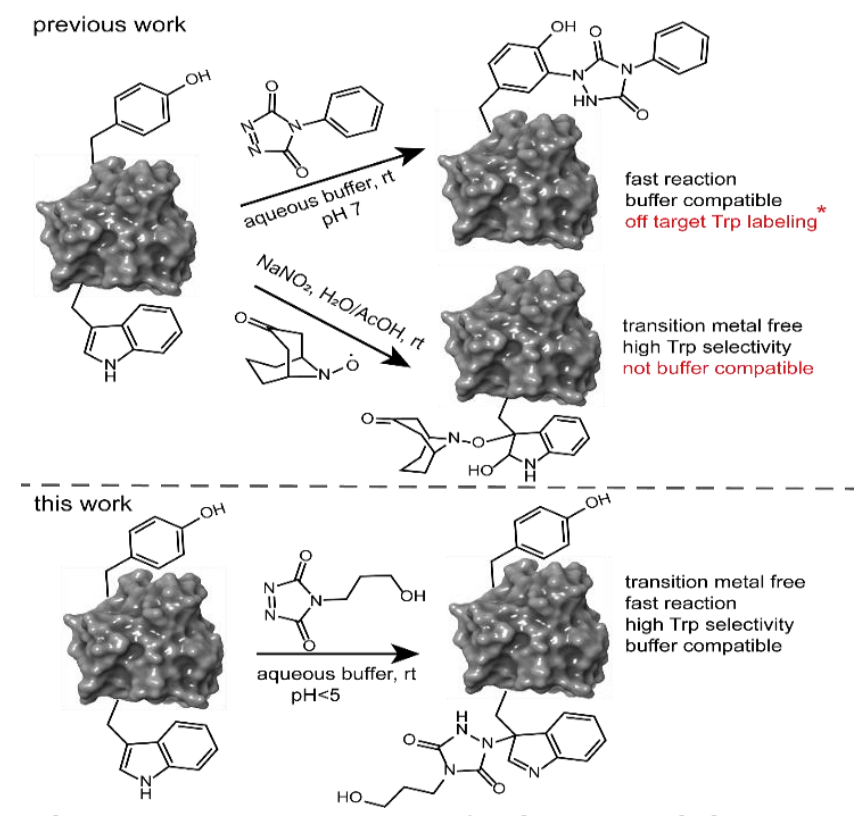

Scheme 1. Prototype reactions for the TAD-Y click, organoradical tryptophan modification (previous work) and TAD tryptophan labeling (this work).

In 2010, Barbas and co-workers reported a click like reaction for the more abundant tyrosine (Tyr, 3.3\% abundance $^{7}$ ) using triazolinedione chemistry, ${ }^{21}$ and several ap- 
plications for protein conjugation followed. $22,23,24,25,26$ Interestingly, when exploring this powerful Tyr click reaction on Trp-containing peptides, we observed a high degree of offtarget labeling on Trp residues, even in aqueous buffers. While the swift reaction of indoles with triazolinediones was reported by Baran, Guerrero and Corey in 2003, ${ }^{27,28}$ Barbas and co-workers demonstrated that tyrosine labeling is kinetically favored in buffers. However, we now surmise that this competitive Trp-labeling in protein bioconjugation remained under the radar, likely due to a combination of the low abundance and low solvent accessibility of Trp residues. Moreover, in line with observations of Baran and coworkers, we found that indole-TAD modifications have limited thermal stability and can reverse under MS/MS conditions rendering their detection more tedious. We thus decided to more closely examine the competition between Trp and Tyr labeling by TADs in order to probe the potential of TAD reagents for selective Trp-bio-conjugation (scheme 1). For that purpose, tetrapeptides NWAS $\mathbf{1 a}$ and NYAS $\mathbf{1 b}$ were tested in intermolecular competition experiments with phenyltriazolinedione (PTAD 2a) in PBS-buffer at two different $\mathrm{pH}$ values, allowing for head to head comparison between Tyr and Trp side chains embedded in the exact same chemical environment (figure 1). Signals for peptide conjugates 2aa and $\mathbf{2 b a}$ overlap on the HPLC UV chromatogram, therefore extracted ion chromatograms (XIC's) were used for the analysis.

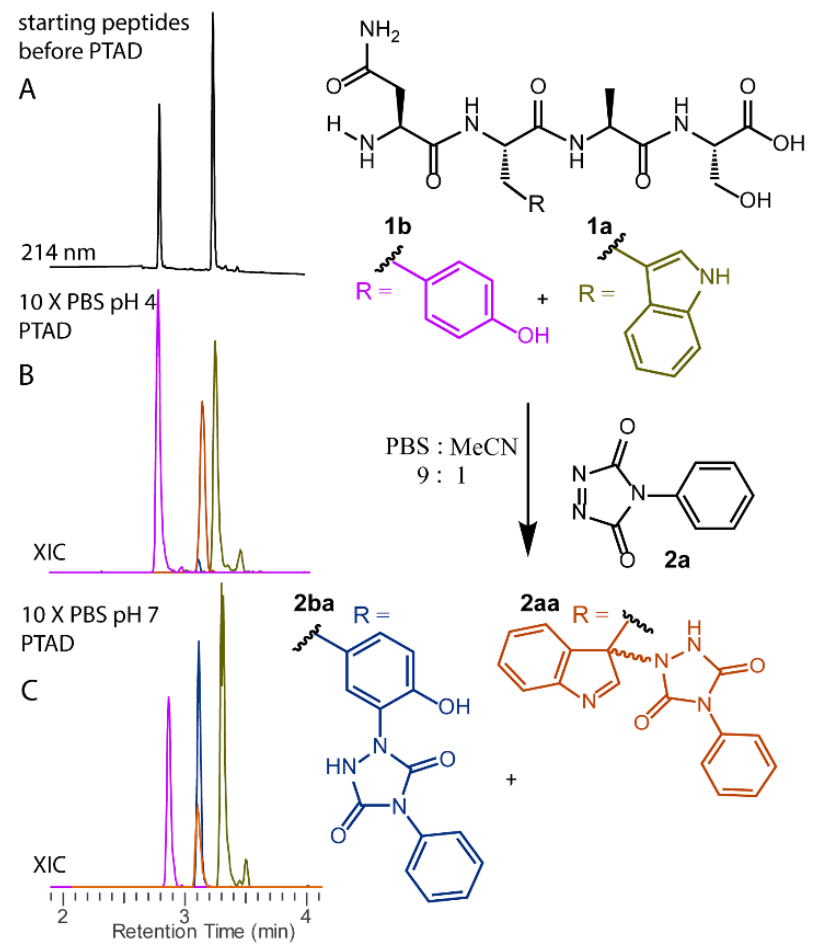

Figure 1. Competition experiment between NWAS (1a, 0.3 $\mathrm{mM}$ ) and NYAS (1b, $0.3 \mathrm{mM}$ ) peptides for PTAD (2a, 0.4 $\mathrm{mM}$ ) in 10x PBS buffers at $\mathrm{pH} 4$ and $\mathrm{pH}$ 7. HPLC chromatogram before reaction (A). Extracted ion chromatograms (XIC) of both starting peptide ions $\mathbf{1 a}$ (green) and $\mathbf{1 b}$ (pink) and conjugated peptide ions 2 aa (orange) and $\mathbf{2 b a}$ (blue) for reaction in 10x PBS at pH 4 (B) or pH 7 (C).
When analyzing the XIC's of the starting peptide-ions NWAS $\mathbf{1 a}$ (green) and NYAS $\mathbf{1 b}$ (pink) and conjugated peptide-ions NWAS-PTAD 2aa (orange) and NYAS-PTAD 2 ba (blue), a pronounced difference can be observed between the reaction at $\mathrm{pH} 4$ and $\mathrm{pH}$ 7. Indeed, at pH 4 Trp conjugate 2aa was detected nearly exclusively while at $\mathrm{pH} 7$ a mixture of conjugates was obtained. This observed $\mathrm{pH}$-dependent reactivity of TADs with Tyr is in accord with previous mechanistic studies of the tyrosine-TAD click reaction, which indicate the phenolate species as the prevalent nucleophile. ${ }^{29}$ Lowering the $\mathrm{pH}$ will effectively decrease the amount of tyrosine-phenolate form and thus decrease the extent of reaction of Tyr with TAD. This was further confirmed using additional peptides (1a-1h, table 1) and TAD-propanol $\mathbf{2 b}$, PTAD-alkyne 2c and fluorescent DMEQ-TAD 2d (Section S2.2.2). It was also observed that, even without competing Trp-peptide present, lowering of $\mathrm{pH}$ causes a significant reduction in Tyr-conjugate formation (Section S2.2.1). These findings at peptide level prompted us to look in more detail to earlier reports on the tyrosine click protein modification. Indeed, off-target Trp-labeling was observed earlier at protein level. In the initial study of Ban et al. modification on tryptophan was observed upon myoglobin labeling albeit in a very low amount. ${ }^{21,25}$ Furthermore, careful reinterpretation of the MALDI-TOF MS spectra (kindly provided by the authors) of Vandewalle et al., ${ }^{23}$ who labeled BSA with butylTAD, showed that Trp-modification was indeed noticeable (Section S3.1). These findings demonstrate that researchers can incorrectly assume that tryptophan will not react with TAD-reagents in protein conjugation reactions, possibly leading to flawed interpretation of data.

Table 1. Peptide sequences used in this study, structures of TAD reagents $2 b, 2 c$ and $2 d$.

\begin{tabular}{ll}
\hline Entry & Sequence \\
\hline $\mathbf{1 a}$ & Asn-Trp-Ala-Ser-OH \\
$\mathbf{1 b}$ & Asn-Tyr-Ala-Ser-OH \\
$\mathbf{1 c}$ & Asn-Ser-Ala-Trp-OH \\
$\mathbf{1 d}$ & Asn-Ser-Ala-Tyr-OH \\
$\mathbf{1 e}$ & Trp-Ser-Ala-Asn-OH \\
$\mathbf{1 f}$ & Tyr-Ser-Ala-Asn-OH \\
$\mathbf{1 g}$ & Lys-Lys-Ser-Tyr-Leu-Ser-Pro-Arg-Thr-Ala-Leu- \\
$\mathbf{1 h}$ & Ile-Asn-Phe-Leu-Val-OH \\
& Lys-Lys-Ser-Trp-Leu-Ser-Pro-Arg-Thr-Ala-Leu- \\
$\mathbf{1 i}$ & Val-Trp-Ser-Asn-Arg-His-Phe-Tyr-OH \\
$\mathbf{1 j}$ & Val-Tyr-Ser-Asn-Arg-His-Phe-Trp-OH \\
$\mathbf{1 k}$ & Val-Trp-Ser-Gln-Lys-Arg-His-Phe-Gly-Tyr-OH \\
$\mathbf{1 l}$ & Lys-Asp-Tyr-Trp-Glu-Cys-Ala-OH \\
\end{tabular}

Triggered by these findings, indicating that a completely Trp-selective modification can be possible, we examined TAD-Trp conjugation in peptides by investigating the influence of the relative amino acid positioning on the outcome of the reaction. Competition experiments between tetrapeptides NWAS 1a and NSAW 1c and TAD-propanol $\mathbf{2 b}$ showed a remarkable difference in reactivity (figure 2). 


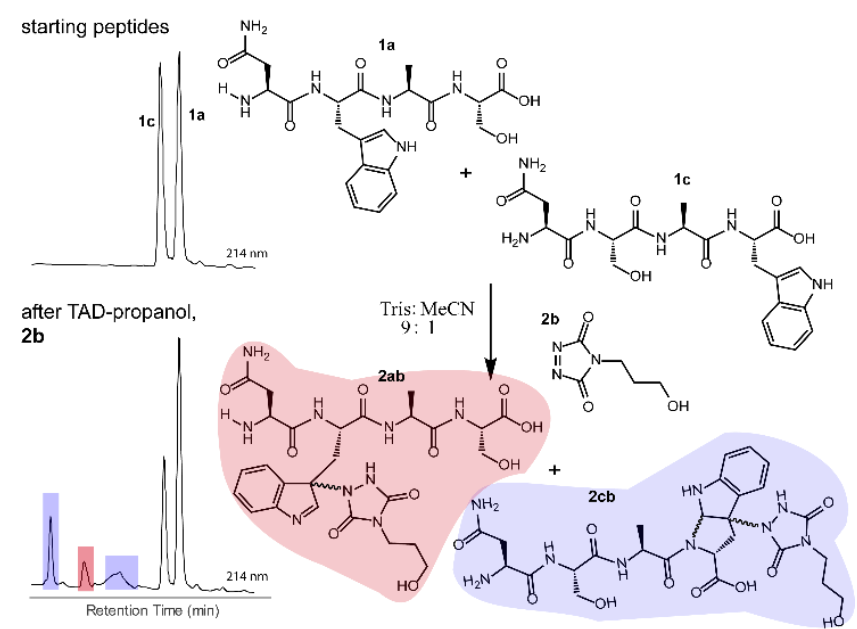

Figure 2. Competition experiment of NWAS (1a, $0.3 \mathrm{mM})$ and NSAW (1c, $0.3 \mathrm{mM}$ ) peptides with TAD-propanol (2b, $0.5 \mathrm{mM}$ ) in Tris buffer pH 5.8. Zoom of the HPLC chromatogram at $214 \mathrm{~nm}$ of the starting mixture (top left) and after reaction with TAD-propanol $\mathbf{2 b}$ (bottom left).

Intermolecular competition between 1a and 1c clearly demonstrates the position-sensitivity of the Trp-TAD reaction: the C-terminal tryptophan in $\mathbf{1 c}$ is labeled to a 3 times higher extent, as calculated via HPLC peak integration at $214 \mathrm{~nm}$, compared to its internal tryptophan 1a counterpart. This reactivity difference can be attributed to the more exposed reactive center as well as to the presence of the carboxylic acid which can transiently donate a proton to the TAD moiety rendering it even more electrophilic. A second striking difference resides in the nature of the formed adducts. For the C-terminal tryptophan, two peaks for the labeled product $\mathbf{2 c b}$ are observed, indicating the formation of isomers. Indeed, we found this adduct had undergone an additional annulation caused by the reaction of the lone pair on the backbone nitrogen with the indole $\mathrm{C} 2$ after reaction of TAD with the indole $\mathrm{C} 3$. These findings were confirmed via NMR analysis of Boc-Trp-OH and N-Ac-Trp-OMe adducts with TAD-propanol $\mathbf{2 b}$ (Section S4) and are in agreement with the results reported by Baran et al. ${ }^{27}$ on non-peptide related TAD-indole reactions.

In a subsequent series of experiments, we investigated if the observed intermolecular selectivity, translates into intramolecular Trp versus Tyr selectivity. To this end, competition experiments were performed with peptides containing both tyrosine and tryptophan (1i-11, table 1). MS/MS analyses were done to determine the modification site. We found that the modification on tryptophan is unstable in all tested MS/MS conditions except for ESI in combination with electron transfer dissociation (ETD). ESI-HCD, ESI-CID as well as MALDI-TOF/TOF all largely lead to the loss of the TAD modification on tryptophan. The TAD modification on tyrosine was found to be stable in all tested conditions. These findings are in agreement with earlier work on the thermoreversibility of indole-TAD reactions. ${ }^{30}$

Peptide VWSQKRHFGY 1k was labeled using TAD-propanol 2b at $\mathrm{pH} 4$ to selectively target the tryptophan residue in the peptide, or at $\mathrm{pH} 7$ to mainly target the tyrosine. The conjugated peptide will have the same mass regardless of the site of the modification (M+: 1464.71), the triply charged precursor (M+++: 488.91) was selected for electron transfer dissociation (ETD) MS/MS analysis. At $\mathrm{pH} 4$ modification only takes place on tryptophan and Trp-modified fragment ions are observed (figure 3 , top). If the modification reaction is performed at $\mathrm{pH} 7$ the major product is the tyrosine modified peptide (figure 3 bottom). However upon detailed analysis of the full ion chromatograms (SI section 2.4.1) it can be seen that in contrast to Trp-labeling which occurs cleanly at $\mathrm{pH} 4$, the Tyr-labeling of this peptide at $\mathrm{pH} 7$ is accompanied by formation of Trp-modified side products. Further confirmation for the off-target labeling at $\mathrm{pH} 7$ is provided through MS based screening for doubly modified peptides (SI, Figure 2.4.2.3) showing double TAD modified peptides only at $\mathrm{pH} 7$, implying additional off-target Trp labeling next to the intended Tyr labeling.

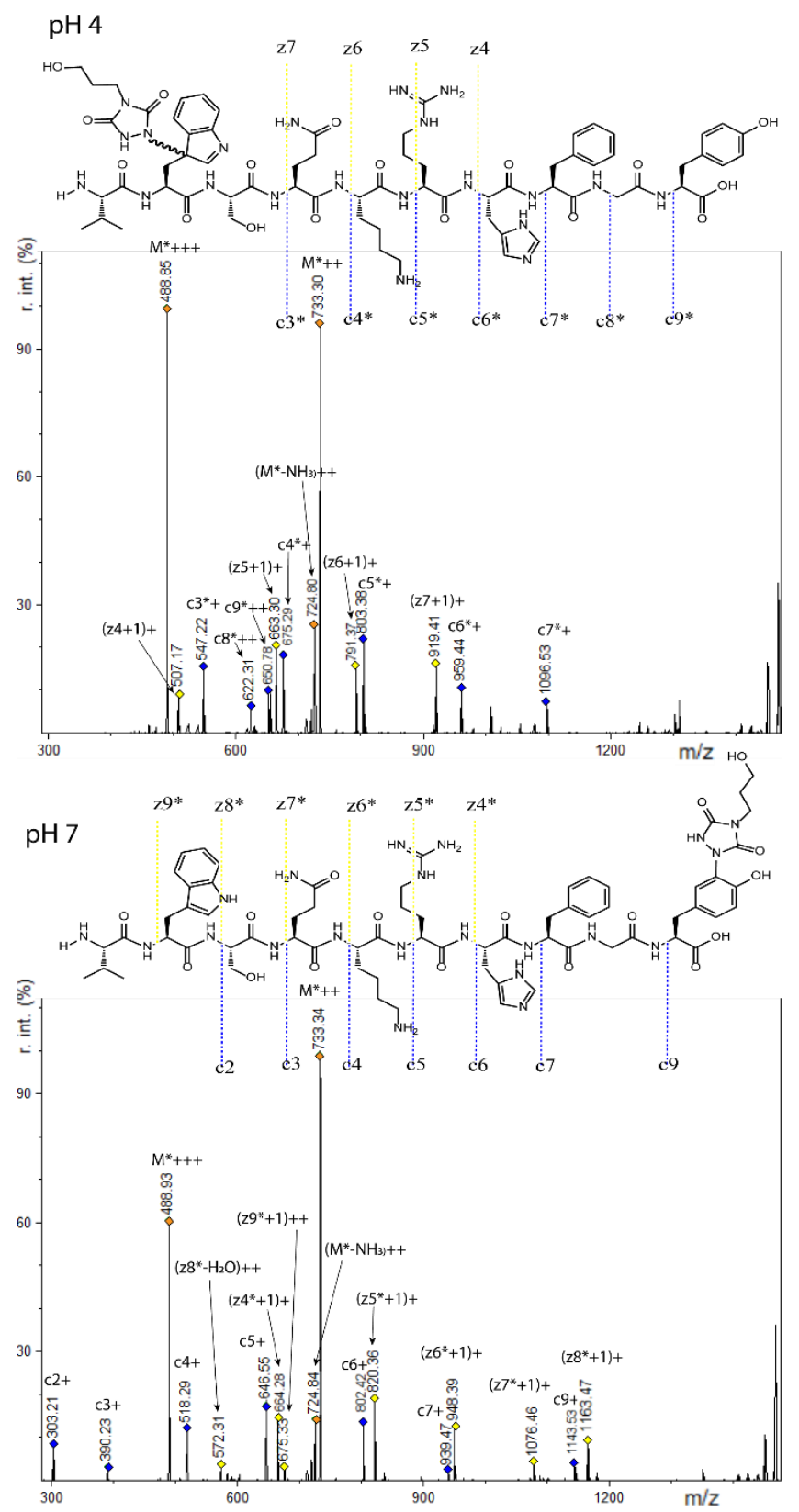

Figure 3. ESI ETD MS/MS of VWSQKRHFGY $\mathbf{1 k}$ after modification with TAD-propanol $\mathbf{2 b}$ in $10 \mathrm{X} \mathrm{PBS} \mathrm{pH} 4$ (top) and pH 7 (bottom). In both MS/MS spectra the chemical structure is depicted with the observed fragment ions. Fragment ions 
corresponding to TAD-modified peptide fragments are indicated with “*”.

We next explored TAD-click reactions for Trp-based protein conjugation. Alphabodies have a triple helical coiled coil structure and are developed for intracellular protein interaction targets by Complix N.V. ${ }^{31}$ Two recombinant alphabodies were used; the valentine alphabody containing no tryptophans and three tyrosines, and alphabody 586D containing one tryptophan residue next to three tyrosines. A competition experiment between the valentine alphabody and tryptophan containing peptide 1c (SI3.2) with $\mathbf{2 b}$ at pH4, resulted in $80 \%$ conversion of $1 \mathrm{c}$ while alphabody conjugation was absent. Protein conjugation with 586D at $\mathrm{pH} 4$ was carried out using fluorescent DMEQ-TAD (2d). MS/MS analysis of the resulting protein conjugate digest (SI3.3) confirms the localization of the TAD modification on tryptophan. Intact protein analysis of the $586 \mathrm{D} 2 \mathbf{d}$ conjugate $(30$ eq., pH 4) shows $60 \%$ conjugation. Together, these results indicate that protein TAD modification can be targeted selectively to a Trp side chain, in the presence of tyrosines. As a negative control the valentine alphabody was reacted with 48 eq. of $\mathbf{2 b}$ at pH 4 and no conjugation was observed.

Finally, human galectin-7,32 containing one tryptophan and one tyrosine residue was treated with $\mathbf{2 b}, \mathbf{2 c}$ and $\mathbf{2 d}$ at $\mathrm{pH}$ 4. The conjugated proteins were observed for all TAD reagents. Intact protein analysis of the galectin-7 $\mathbf{2 d}$ conjugate (10 eq., pH 4, SI3.4) shows over $50 \%$ conjugation. Analyses of the galectin-7 TAD propanol conjugate digests confirm the localization of the TAD moiety on tryptophan. Furthermore the MS/MS analyses of conjugation experiments with 2b (20 eq.; $\mathrm{pH} 4$ and 7) demonstrate that at $\mathrm{pH} 4$ the tryptophan has almost exclusively reacted with TAD while at $\mathrm{pH}$ 7 both the tyrosine and the tryptophan had reacted. Additionally, in accord with the findings on the peptide level we found the TAD modification on tryptophan to be labile under the HCD MS/MS conditions used in these experiments.

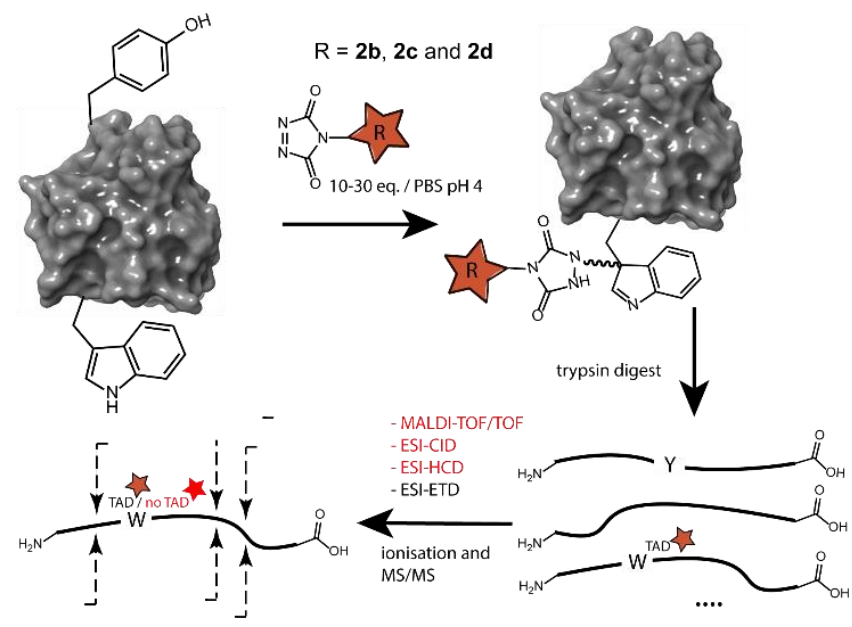

Figure 4. Schematic representation of protein conjugation reaction of alphabody 586D and galectin-7 with $2 \mathbf{2 b}, \mathbf{2 c}$ and 2d, subsequent trypsin digest followed by different ionization and MS/MS fragmentation of the modified tryptic fragment. Depending on the applied technique the TAD modification on $\mathrm{W}$ can be observed or not.

In conclusion, we report that competitive tryptophan labeling is liable to have so far been systematically overlooked in the current use of triazolinedione (TAD) chemistry for putative tyrosine-selective protein conjugation, a technique which is growing in popularity. The reversibility of the TADtryptophan in MS/MS analysis, in combination with the low abundance and low accessibility of tryptophan side chains likely caused this off-target effect to have remained under the radar. We have found that an exposed tryptophan is in fact kinetically favored over tyrosine in most conditions. Lowering the buffer $\mathrm{pH}$ further enhanced the selectivity resulting in a transition metal free, buffer-compatible amino acid specific labeling method for the least abundant natural amino acid tryptophan. Thus, in addition to a better understanding of the factors that govern the click-like TAD-based protein conjugation, its scope has been expanded, and a very interesting new option for native amino acid selective modification has been revealed. The implementation of Trpsubstitutions at protein surfaces or loops can thus be an interesting rational design strategy for fully site-selective labeling of native proteins.

\section{ASSOCIATED CONTENT}

Supporting information: this material is available free of charge via the Internet.

Supplementary methods and figures S1.2.1 - S4.2.1 Synthesis and characterization of TAD derivatives, peptides and peptide conjugates.

Synthesis and characterization of protein conjugates. NMR spectra

\section{AUTHOR INFORMATION}

\section{Corresponding Author}

Annemieke Madder - Organic and Biomimetic Chemistry Research group OBCR, Department of Organic and Macromolecular Chemistry, Ghent University, 9000 Ghent, Belgium; orcid.org/0000-0003-0179-7608; E-mail: annemieke.madder@ugent.be

\section{Authors}

Klaas Decoene - Organic and Biomimetic Chemistry Research group OBCR, Department of Organic and Macromolecular Chemistry, Ghent University, 9000 Ghent, Belgium.

Kamil Unal - Organic Synthesis group, Department of Organic and Macromolecular Chemistry, Ghent University, 9000 Ghent, Belgium.

An Staes - VIB Center for Medical Biotechnology, Technologiepark-Zwijnaarde 75, 9052 Ghent, Belgium; Department of Bio-molecular Medicine, Ghent University, TechnologieparkZwijnaarde 75, 9052 Ghent, Belgium \& VIB core facility, VIB Center for Medical Biotechnology, Technologiepark-Zwijnaarde 75, 9052 Ghent, Belgium; orcid.org/0000-0001-8767-8508; Email: An.Staes@vib-UGent.be

Kris Gevaert - VIB Center for Medical Biotechnology, Technologiepark-Zwijnaarde 75, 9052 Ghent, Belgium \& Department of Bio-molecular Medicine, Ghent University, TechnologieparkZwijnaarde 75, 9052 Ghent, Belgium; orcid.org/0000-00024237-0283; E-mail: Kris.Gevaert@UGent.be

Johan Winne - Organic Synthesis group, Department of Organic and Macromolecular Chemistry, Ghent University, 9000 Ghent, Belgium; orcid.org/0000-0002-9015-4497; E-mail: johan.winne@ugent.be

Author Contributions 
The manuscript was written through contributions of all authors. / All authors have given approval to the final version of the manuscript.

\section{Notes}

The authors declare no competing financial interests.

\section{ACKNOWLEDGMENT}

The FWO Vlaanderen is gratefully acknowledged for financial support (grant agreement G.0485.16N). K.U. acknowledges UGent BOF for funding. Complix N.V. is acknowledged for the supply of the alphabodies used in this study.

\section{REFERENCES}

(1) Hoyt, E. A.; Cal, P. M. S. D.; Oliveira, B. L.; Bernardes, G. J. L. Contemporary Approaches to Site-Selective Protein Modification. Nat. Rev. Chem. 2019, 3 (3), 147-171.

(2) Krall, N.; Da Cruz, F. P.; Boutureira, O.; Bernardes, G. J. L. L. Site-Selective Protein-Modification Chemistry for Basic Biology and Drug Development. Nat. Chem. 2016, 8 (2), 103113.

(3) Agarwal, P.; Bertozzi, C. R. Site-Specific Antibody-Drug Conjugates: The Nexus of Bioorthogonal Chemistry, Protein Engineering, and Drug Development. Bioconjug. Chem. 2015, 26 (2), 176-192.

(4) Bottecchia, C.; Noël, T. Photocatalytic Modification of Amino Acids, Peptides, and Proteins. Chemistry - A European Journal. Wiley-VCH Verlag January 2, 2019, pp 26-42.

(5) Baslé, E.; Joubert, N.; Pucheault, M. Protein Chemical Modification on Endogenous Amino Acids. Chem. Biol. 2010, 17 (3), 213-227.

(6) Gunnoo, S. B.; Madder, A. Chemical Protein Modification through Cysteine. ChemBioChem 2016, 17 (7), 529-553.

(7) https://www.uniprot.org/statistics/Swiss-Prot.

(8) deGruyter, J. N.; Malins, L. R.; Baran, P. S. Residue-Specific Peptide Modification: A Chemist's Guide. Biochemistry 2017, 56 (30), 3863-3873.

(9) Yu, Y.; Zhang, L. K.; Buevich, A. V.; Li, G.; Tang, H.; Vachal, P.; Colletti, S. L.; Shi, Z. C. Chemoselective Peptide Modification via Photocatalytic Tryptophan $\beta$-Position Conjugation. J. Am. Chem. Soc. 2018, 140 (22), 6797-6800.

(10) Kaplaneris, N.; Rogge, T.; Yin, R.; Wang, H.; Sirvinskaite, G.; Ackermann, L. Late-Stage Diversification through Manganese-Catalyzed C-H Activation: Access to Acyclic, Hybrid, and Stapled Peptides. Angew. Chemie 2019, 131 (11), 3514-3518.

(11) Wang, W.; Lorion, M. Ø. M.; Shah, J.; Kapdi, A. R. Late-Stage Peptide Diversification by Position-Selective C À H Activation Angewandte. 2018, 14700-14717.

(12) Tolnai, G. L.; Brand, J. P.; Waser, J. Gold-Catalyzed Direct Alkynylation of Tryptophan in Peptides Using TIPS-EBX. Beilstein J. Org. Chem. 2016, 12, 745-749.

(13) Ruan, Z.; Sauermann, N.; Manoni, E.; Ackermann, L. Manganese-Catalyzed C-H Alkynylation: Expedient Peptide Synthesis and Modification. Angew. Chemie Int. Ed. 2017, 56 (12), 3172-3176.

(14) Schischko, A.; Ren, H.; Kaplaneris, N.; Ackermann, L. Bioorthogonal Diversification of Peptides through Selective Ruthenium(II)-Catalyzed C-H Activation. Angew. Chemie - Int. Ed. 2017, 56 (6), 1576-1580.

(15) Hansen, M. B.; Hubálek, F.; Skrydstrup, T.; Hoeg-Jensen, T. Chemo- and Regioselective Ethynylation of TryptophanContaining Peptides and Proteins. Chem. - A Eur. J. 2016, 22
(5), 1572-1576.

(16) Ruiz-Rodriguez, J.; Albericio, F.; Lavilla, R. Postsynthetic Modification of Peptides: Chemoselective C-Arylation of Tryptophan Residues. Chem. - A Eur. J. 2010, 16 (4), 11241127.

(17) Malins, L. R.; Cergol, K. M.; Payne, R. J. Chemoselective Sulfenylation and Peptide Ligation at Tryptophan. Chem. Sci. 2014, 5 (1), 260-266.

(18) Antos, J. M.; McFarland, J. M.; Iavarone, A. T.; Francis, M. B. Chemoselective Tryptophan Labeling with Rhodium Carbenoids at Mild PH. J. Am. Chem. Soc. 2009, 131 (17) 6301-6308.

(19) Seki, Y.; Ishiyama, T.; Sasaki, D.; Abe, J.; Sohma, Y.; Oisaki, K Kanai, M. Transition Metal-Free Tryptophan-Selective Bioconjugation of Proteins. J. Am. Chem. Soc. 2016, 138 (34), 10798-10801.

(20) Tower, S. J.; Hetcher, W. J.; Myers, T. E.; Kuehl, N. J.; Taylor, M. T. Selective Modification of Tryptophan Residues in Peptides and Proteins Using a Biomimetic Electron Transfer Process. J. Am. Chem. Soc. 2020, jacs.0c03039.

(21) Ban, H.; Gavrilyuk, J.; Barbas, C. F. Tyrosine Bioconjugation through Aqueous Ene-Type Reactions: A Click-like Reaction for Tyrosine. J. Am. Chem. Soc. 2010, 132 (5), 1523-1525.

(22) Ban, H.; Nagano, M.; Gavrilyuk, J.; Hakamata, W.; Inokuma, T.; Barbas, C. F. Facile and Stabile Linkages through Tyrosine: Bioconjugation Strategies with the Tyrosine-Click Reaction. Bioconjug. Chem. 2013, 24 (4), 520-532.

(23) Vandewalle, S.; De Coen, R.; De Geest, B. G.; Du Prez, F. E Tyrosine-Triazolinedione Bioconjugation as Site-Selective Protein Modification Starting from RAFT-Derived Polymers. ACS Macro Lett. 2017, 1368-1372.

(24) Alvarez-Dorta, D.; Thobie-Gautier, C.; Croyal, M.; Bouzelha, M.; Mével, M.; Deniaud, D.; Boujtita, M.; Gouin, S. G. Electrochemically Promoted Tyrosine-Click-Chemistry for Protein Labeling. J. Am. Chem. Soc. 2018, 140 (49), 17120 17126.

(25) Hu, Q.-Y.; Allan, M.; Adamo, R.; Quinn, D.; Zhai, H.; Wu, G.; Clark, K.; Zhou, J.; Ortiz, S.; Wang, B.; et al. Synthesis of a WellDefined Glycoconjugate Vaccine by a Tyrosine-Selective Conjugation Strategy. Chem. Sci. 2013, 4 (10), 3827.

(26) Moinpour, M.; Barker, N.; Guzman, L.; Jewett, J.; Langlais, P.; Schwartz, J. Determining Protein Structure by Tyrosine Bioconjugation. Protein Sci. 2020.

(27) Baran, P. S.; Guerrero, C. A.; Corey, E. J. The First Method for Protection-Deprotection of the Indole 2,3- $\pi$ Bond. Org. Lett. 2003, 5 (11), 1999-2001.

(28) Hanay, S. B.; Brougham, D. F.; Dias, A. A.; Heise, A. Investigation of the Triazolinedione (TAD) Reaction with Tryptophan as a Direct Route to Copolypeptide Conjugation and Cross-Linking. Polym. Chem. 2017, 8 (43), 6594-6597.

(29) Kaiser, D.; Winne, J. M.; Ortiz-Soto, M. E.; Seibel, J.; Le, T. A.; Engels, B. Mechanistical Insights into the Bioconjugation Reaction of Triazolinediones with Tyrosine. J. Org. Chem. 2018, 83 (17), 10248-10260.

(30) Billiet, S.; De Bruycker, K.; Driessen, F.; Goossens, H.; Van Speybroeck, V.; Winne, J. M.; Du Prez, F. E. Triazolinediones Enable Ultrafast and Reversible Click Chemistry for the Design of Dynamic Polymer Systems. Nat. Chem. 2014, 6 (9), 815-821.

(31) Desmet, J.; Verstraete, K.; Bloch, Y.; Lorent, E.; Wen, Y.; Devreese, B.; Vandenbroucke, K.; Loverix, S.; Hettmann, T. Deroo, S.; et al. Structural Basis of IL-23 Antagonism by an Alphabody Protein Scaffold. Nat. Commun. 2014, 5 (1), 5237.

(32) Saussez, S.; Kiss, R. Galectin-7. Cell. Mol. Life Sci. 2006, 63 (6), 686-697. 